\title{
TIME-DEPENDENT STUDIES OF THE SETTLING OF HEAVY ELEMENTS IN THE ENVELOPES OF COOL WHITE DWARFS
}

\author{
J. Dupuis, C. Pelletier, G. Fontaine, and F. Wesemael \\ Département de Physique, Université de Montréal
}

Gravitational settling is widely accepted as being a fundamental physical process acting upon superficial layers of white dwarfs and resulting in an important alteration of their atmospheric composition. Several investigators have been interested by the problem of gravitational settling in white dwarfs (Fontaine and Michaud 1979; Vauclair, Vauclair, and Greenstein 1979; Alcock and Ilarianov 1980; Muchmore 1984; Paquette et al. 1986). As pointed out in Paquette et al. 1986, they all reached the same qualitative conclusion: the gravitational settling time scales of metals in cool white dwarfs are small compared to their evolutionary time scales. These stars should therefore have their photospheres depleted of metals if there is no extrinsic source such as accretion for example. This is consistent with the observational fact that most of the cool white dwarfs spectra just show hydrogen and helium lines while the absence of metallic lines indicates a strong depletion of metals. Although the qualitative agreement between theory and observations is satisfactory, only time-dependent calculations can lead to a thorough understanding of the heavy element abundance patterns in cool white dwarfs. In particular, the predicted abundance of an element within the framework of the accretion-diffusion model does depend explicitly on the results of such calculations. We have already presented some preliminary results of numerical simulation of accretion episodes of heavy elements into white dwarfs (Dupuis et al. 1987). As part of an ongoing detailed investigation of these processes, we focus here exclusively on the mechanism of gravitational settling in white dwarfs in order to clear some confusion which has appeared in the literature.

The usual notion of an $e$-folding diffusion time scale strictly applies at the base of the convection zone of a stellar model after having made a number of approximations. If an element can be considered as trace and if ordinary diffusion can be neglected (i.e. the concentration gradient is much smaller than its equilibrium value; see Fontaine and Michaud 1979), then it is a simple matter to show that, at the base of a convection zone, the abundance of a given element is given by,

$$
u=u_{0} \exp (-t / \theta)
$$

where $\theta$, the diffusion or settling time scale, is given by,

$$
\theta=\frac{g}{4 \pi G} \frac{q}{\rho|\omega|}
$$

where $G$ is the gravitational constant, $g$ is the local gravity, $q$ is the fractional mass, $\rho$ is the density, and $\omega$ is the diffusion velocity of the trace element with respect to the background $(\omega<0)$. The exponential behavior shown in equation (1) is not valid in regions below the convection zone and the notion of an $\boldsymbol{e}$-folding diffusion time scale loses its significance there. The time-dependence of the abundance at a given shell can only be obtained from a solution of the continuity equation and, generally, the behavior is very different from an exponential 
behavior. We illustrate the point here with a few sample results. The diffusion equation for gravitational settling is solved with a new technique based on a Galerkin-type finite element formulation briefly described in Pelletier et al. (1988). We consider the settling of a typical heavy element, namely calcium, in the envelopes of $H e$ rich white dwarf models with $T_{e} \leq 25,000 \mathrm{~K}$. For a given model, we have set the initial calcium abundance equal to its solar value $\left(u(\mathrm{Ca})=5.96 \times 10^{-6}\right)$ throughout the envelope. We have then followed the evolution of the abundance profile during an interval of time equal to the age of the model. In Figure 1, the result of such a simulation is shown for calcium diffusing in a 10,000 K, $0.6 M_{\odot} H e$-rich white dwarf having a $H e$ layer mass $\log \Delta M(H e) / M=-3.5$ and computed with the standard treatment of convection. Each curve gives the logarithmic abundance of calcium as a function of the logarithmic mass fraction for increasing integration time from top to bottom. First, one should notice that the calcium abundance profile evolution is followed during more than $9 \times 10^{8}$ years and that the surface abundance (the value at $\log q \approx-6.2$ ) varies almost over 25 orders of magnitude without significant loss of precision. Such a simulation required 170 cubic elements and took 331 time steps, which means about $160 \mathrm{CPU}$ seconds of execution time on the Université de Montréal's CYBER 855. A noteworthy feature is that the overall mass of calcium contained in the envelope is conserved to better than 1 $\%$. In Figure 2, we show the logarithmic abundance of calcium as a function of logarithmic time in unit of years for 8 different layers in the envelope. The curve labelled 1 is the solution at the surface of the envelope which is delimited by the bottom of the superficial $H e$ convection zone, and the 7 remaining curves labelled 2 to 8 are the solution at increasingly deeper layers separated by approximately half a dex in mass. The continuous curves are the numerical solutions while the dotted curves are the exponential solutions defined by the local diffusion time scales as discussed above. The surface abundance (curve 1) is falling as expected almost exponentially with time, the increasing difference with the exponential solution is due to the sligth buildup of the concentration gradient which slows down the settling of calcium. From a practical and observational point of view, this result is not different from those of previous studies: calcium falls under its detection level in an interval of time small compared to the age of the model (at $T_{e}=10^{4} \mathrm{~K}$, age $=9 \times 10^{8}$ years). This can also be understood with the help of figure 1 , the calcium profile near the surface relaxes rapidly during the first $10^{7}$ years where the concentration gradient is negligibly small. After $10^{7}$ years, settling tends to be opposed by the concentration gradient which causes the deviation from the exponential solution to become even more important on curve 1 of figure 2 .

The behavior of the solution in deeper layers is quite different from the exponential solution and is understood in the following way: before a time about equal to the local settling time scale, the local abundance exceeds the exponential solution values because calcium coming from overlying layers is causing a temporary enrichment. Thereafter, the depletion in calcium in the overlying layers starts being felt and the local calcium abundance is getting smaller than the predicted exponential abundance. Finally, when the concentration gradient sets in, the settling is slowed down and the calcium abundance becomes greater than the exponential solution. These results show that the exponential solution associated to the local settling time scale is a good approximation of the surface behavior but that it is not even a good order of magnitude estimate of the solution in deeper layers. This illustrates the need for complete time-dependent studies of gravitational settling since simple $e$-folding time scale estimates are not good approximations of the real solution.

In Figure 3, the logarithmic surface abundance of calcium is shown as a function of logarithmic time (years) for models with effective temperatures ranging from $2.5 \times 10^{4} \mathrm{~K}$ to $8 \times 10^{3} \mathrm{~K}$. For the five models shown, the surface 


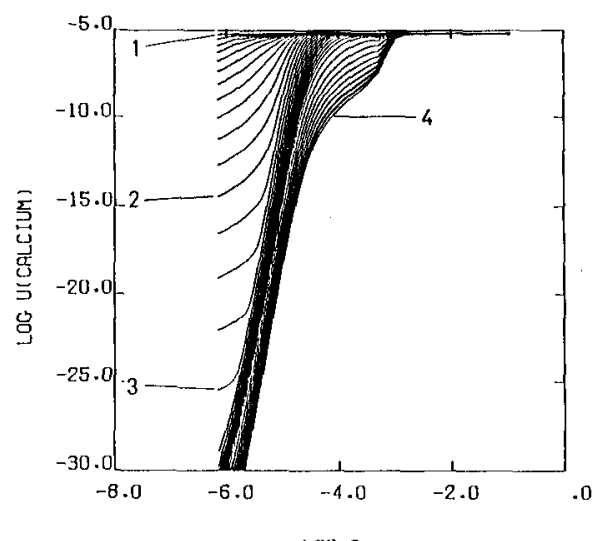

LOG 0

Fig. 1. Evolution of the $C a$ abundance profile in a $10,000 \mathrm{~K}, 0.6 M_{\odot}, H e$-rich white dwarf $(\log q(H e)=$ $-3.5)$ with $M L 1$ convection. The curves labelled 1 to 4 are respectively the profiles after $1.67 \times 10^{4}, 4.1 \times 10^{6}$, $1.01 \times 10^{7}$, and $9.01 \times 10^{8}$ years of settling.

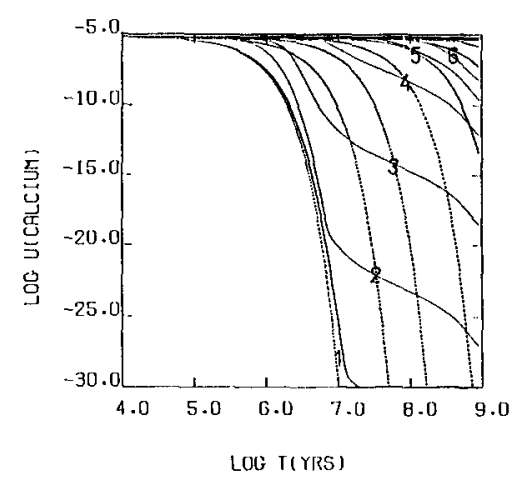

Fig. 2. Evolution of the $C a$ abundance at 6 depths in the envelope of the model of fig. 1 . The continuous and dotted curves are respectively the numerical and exponential solutions. Curves 1 to 6 are associated to layers of $\log \Delta M / M=-6.14,-5.5,-5 .,-4.5,-4 .,-3.5$.
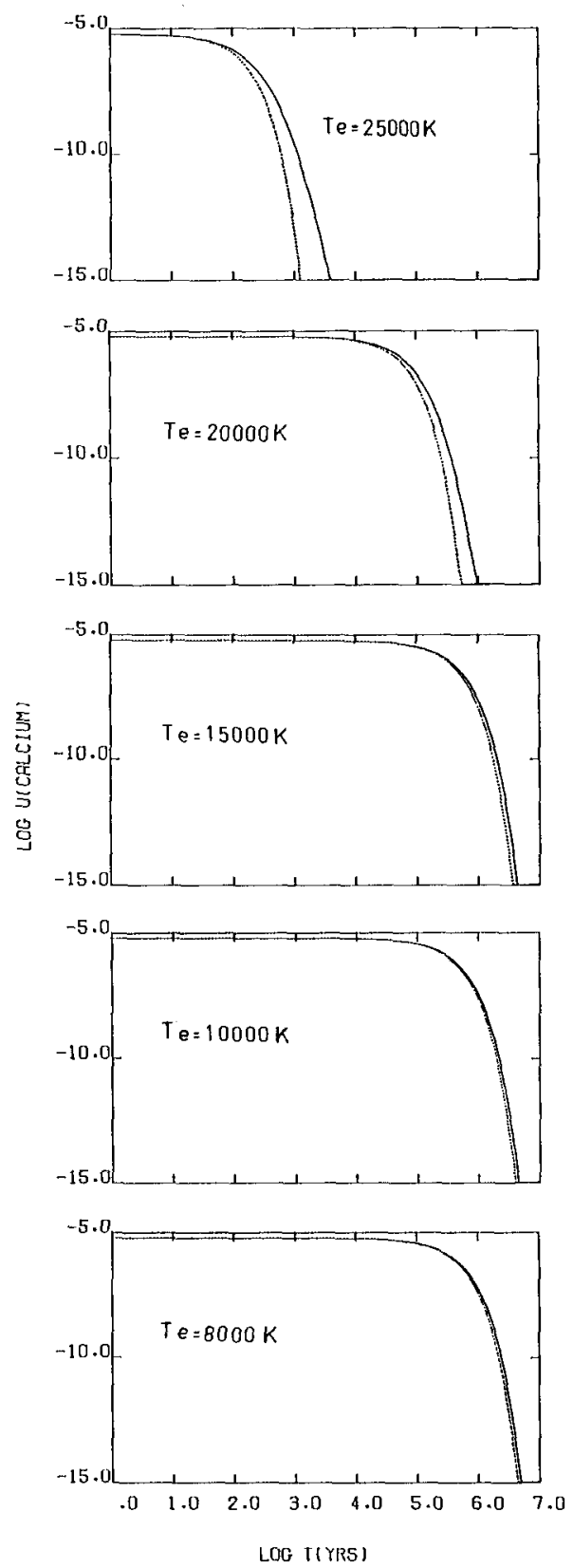

Fig. 3. Surface abundance as a function of time in $H e$-rich white dwarfs with $T_{e}$ ranging from 25,000 to $8,000 \mathrm{~K}$. The continuous and dotted curves represent similar solutions as in fig. 2. 
solution behaves almost exponentially as expected but with a departure from the pure exponential solution which is clearly increasing with effective temperature. This temperature-dependent departure is first explained by the fact that the diffusion time scales are smaller for the hotter models implying that local equilibrium could be reached faster. Also, the equilibrium gradient are smaller for hotter models which makes the equilibrium easier to reach. At equilibrium, the tail of the distribution goes as $u \propto q^{\beta}$ where $\beta=\left(1+Z_{1}\right) A_{2} / A_{1}-Z_{2}-1$ (Pelletier et al. 1986). The quantity $\beta$ gets smaller in hotter models since the base of the convection zone is upper in the envelope thus implying smaller ionization of both the main species $\left(Z_{1}\right)$ and the trace element $\left(Z_{2}\right)$ and therefore smaller equilibrium gradients.

Another unexpected result of time-dependent studies of gravitational settling is that heavy elements do not sink into the deep core of a white dwarf as could be naively expected. Instead, the heavy elements accumulate at the base of the envelope. This behavior is clearly seen in figure 1 where calcium has not been depleted for $\log q \geq-3$. This can be explained as follows: the diffusion equation can be written (Pelletier et al. 1986),

$$
\omega_{12}=D_{12}(1+\gamma)\left[-\frac{\partial \ln C_{2}}{\partial r}+\left(\frac{A_{1} Z_{2}-A_{2} Z_{1}}{Z_{1}+\gamma Z_{2}}\right) \frac{m_{p} g}{k T}+\left(\frac{Z_{2}-Z_{1}}{Z_{1}+\gamma Z_{2}}\right) \frac{\partial \ln P_{i}}{\partial r}\right]
$$

where the first term corresponds to ordinary diffusion and the second and third terms are responsible for gravitational settling. Deep in the envelope, the trace element can be almost completly pressure ionized and that makes the factor $\left(A_{1} Z_{2}-A_{2} Z_{1}\right)$ very small. In addition, when the plasma is degenerate, the third term also becomes small since the temperature and density distribution are close to being uniform thus making the ionic pressure gradient ( $\partial \ln P_{i} / \partial r$ ) also small. The interesting consequence is to slow down and then stop settling in these layers which creates a significant accumulation of heavy elements. Such an accumulation zone could affect the conductive opacity by an increase in the number of highly ionized electron scatterers, thus slowing down the rate at which heat is flowing toward the outer layers. It remains to be seen whether or not this effect is sufficiently large to slow down significantly the cooling rates of white dwarfs. We are exploring more carefully this interesting possibility.

This research has been supported by the NSERC Canada, the fund FCAR (Québec) and a E.W.R. Steacie Fellowship to one of us (GF).

\section{References}

Alcock, C. and Illarionov, A. 1980, Astrophys. J., 235, 534.

Dupuis, J., Pelletier, C., Fontaine, G., and Wesemael, F. 1987, in IAU Colloquium 95, The Second Conference on Faint Blue Stars, eds. A.G.D. Philip, D.S. Hayes, and J. Liebert (Schenectady: L. Davis Press), p. 657.

Fontaine, G., and Michaud, G. 1979, Astrophys. J., 231, 826.

Paquette, C., Pelletier, C., Fontaine, G., and Michaud, G. 1986, Astrophys. J. Suppl., 61, 197.

Pelletier, C., Fontaine, G., Wesemael, F., Michaud, G., and Wegner, G. 1986, Astrophys. J., 307, 242.

Pelletier, C., Fontaine, G., and Wesemael, F. 1988, these proceedings.

Vauclair, G., Vauclair, S., and Greenstein, J. L., 1979, Astron. Astrophys., 155, 356. 